\title{
Maxillofacial infection. A retrospective evaluation of eight years
}

\author{
Bruno Veronez ${ }^{1}$, Fernando Pando de Matos $^{1}$, Marcelo Silva Monnazzi ${ }^{1}$, Alexander Tadeu Sverzut ${ }^{1}$, \\ Cássio Edvard Sverzut ${ }^{1}$, Alexandre Elias Trivellato ${ }^{1}$
}

${ }^{1}$ Universidade de São Paulo - USP, Dental School of Ribeirão Preto, Department of Oral and Maxillofacial Surgery and Periodontology, Ribeirão Preto, SP, Brazil

Received for publication: March 14, 2014 Accepted: April 16, 2014

Correspondence to: Marcelo Silva Monnazzi, Rua Voluntários da Pátria, 2777, CEP: 14801-320 AP 1001, Araraquara, SP, Brasil Phone: + 551633845822

E-mail: monnazzi@ig.com.br

\begin{abstract}
Aim: To evaluate medical records from patients who had maxillofacial infections between August 2002 and May 2010. Methods: Analysis of 157 patients' data looking for epidemiology, causes of the infection, type of treatment established and complications. Results: 113 patients had odontogenic infections and 44 had non-odontogenic infections. The white ethnicity was the most affected (64.33\%), prevailing the men (53.5\%). The odontogenic infections occurred mostly at the mandible (55.74\%), involving the posterior teeth (82\%). Dental decay was the main etiology (90.90\%). The most affected facial anatomic region was the submandibular (42.85\%). Surgical treatment was required in $76 \%$ of the cases. Conclusions: Maxillofacial infections should be treated as soon as possible. Even without culture and antibiogram results, it was possible to treat the infection and to reestablish tissue function.
\end{abstract}

Keywords: focal infection, dental; epidemiology; retrospective studies.

\section{Introduction}

Maxillofacial infections are not rare and could be considered as a public health problem due to their great potential of spreading to important and vital anatomical structures, such as the respiratory system and mediastinum, increasing the risk of septicemia and death for the affected patients ${ }^{1-2}$.

Usually those infections are limited and easily treated ${ }^{3}$. However, there are cases of fatal outcomes due to airway obstruction, since they can progress rapidly if not adequately treated ${ }^{3}$. Maxillofacial infections (MI) are characterized as polymicrobial, endogenous, opportunistic, dynamical and mixed (aerobic and anaerobic bacteria $)^{4}$.

The literature shows that MI affects mainly male patients either in adult or child populations. The odontogenic infection (OI) has multiple possible triggering factors. It could be related to dental decay, periapical and/or periodontal abscess, pericoronitis, pulpitis, osteitis, apart from others ${ }^{5-7}$. Peterson et al. $(2002)^{3}$ point out two major causes for the OI, the periapical (due to pulp necrosis and subsequent bacterial invasion) and the periodontal (as a result of periodontal disease) that allows inoculation of bacteria into deep tissues.

Studies concerning the facial infections are needed to evaluate the treated population and to upgrade whenever required the initial evaluation and treatment in order to improve the results. These studies are also an important contribution to the expertise education ${ }^{8-9}$.

The aim of this study was to evaluate retrospectively the epidemiology, treatment and complications of the MI in patients who presented odontogenic or 
non-odontogenic infections in hospitals treated by our team of surgeons in Ribeirão Preto, SP, Brazil, between August 2002 and May 2010.

\section{Material and methods}

The present epidemiologic survey was carried out by collecting data from patient records in the Department of Oral and Maxillofacial Surgery of Ribeirão Preto Dental School of the São Paulo University - USP. The collected data ranged from August 2002 to May 2010. This research was approved by the Ribeirão Preto Dental School (USP) Ethics Committee under the registration number: 2011.1.177.58.2.

The medical records of all patients who presented MI of odontogenic or nonodontogenic cause during the aforementioned period were revised and included in this study, without any kind of distinction. The only exclusion criteria were incorrect or incomplete records.

Medical records of 157 patients were evaluated. The following data were collected: gender, age, race, first evaluation date, intervention date, medical history, addictions, dentition, oral hygiene, etiology, signs and symptoms, affected facial regions, adopted therapeutics, discharge date and complications, such as dehiscence, need for reintervention or medication change and residual scars.

For patients whose treatment required surgical intervention, it was performed by the following procedures: removal of the etiologic agent, puncture and aspiration of the affected area, incision and drainage of the cellulitis and/ or abscess, necrotic tissues debridement and abundant irrigation with saline. Penrose and irrigation drain insertion was done and the drains were kept for at least $48 \mathrm{~h}$. The harvested secretion was sent for microbiological analysis for all patients who required surgical intervention, but all culture and antibiogram results were negative. Empiric antibiotic therapy was prescribed for all patients. The first choice for antibiotic prescription was amoxicillin associated to clavulanate with the dosage of $1 \mathrm{~g}$ every $6 \mathrm{~h}$, by intravenous administration during the hospital stay. However, in the initial years of this study were found records describing the use of cephalothin associated to metronidazole, by intravenous administration every 6 and $8 \mathrm{~h}$ respectively, because the amoxicillin/clavulanate was not a standard drug at the hospitals back then

Preoperative image exams were obtained providing radiographic diagnosis for the infection cause; the main exams were the postero-anterior mandible radiographs and in more severe cases computed tomography scans were provided. Surgically treated patients received saline irrigation through irrigation drains previously inserted at the infection site, twice a day until secretion was reduced. These patients were discharged after the involution of the infectious signs and symptoms and improvement of the systemic medical conditions. At discharge time the drains were removed and the patient maintained the use of antibiotic medications by oral administration until completing seven days after surgery
The follow-up protocol was weekly until completing one month postoperatively, and after that it was scheduled once every month, for at least 6 months. However, this protocol was not followed in all cases, because some patients did not return.

The data were collected by a single examiner and tabulated for analysis in Microsoft Access ${ }^{\circledR} 2007$ software, then the data were shown in tables and a descriptive analysis of the results was done. The authors also state having read the Helsinki Declaration and following its guidelines in this investigation, and that none has any kind of conflict of interest.

\section{Results}

The medical record data collection revealed that, out of the 157 patients that were affected by facial infection, 113 cases had odontogenic cause $(72.15 \%)$ and 44 patients presented nonodontogenic infection $(27.85 \%)$. Men were the most affected $(53.5 \%)$, while women were less affected $(46.50 \%)$. Caucasians were more affected by the facial infection $(64.33 \%)$, followed by the brown $(23.41 \%)$, black $(7.64 \%)$ and Asian $(0.63 \%)$ populations. The records revealed $3.99 \%$ files without ethnicity data.

When the odontogenic infections were evaluated regarding the affected dental arch, it was verified that the mandibular arch was more affected $(55.73 \%)$ in comparison to the maxillary arch $(44.26 \%)$. The permanent dentition was more affected by the infectious process $(87.34 \%)$, followed by the primary $(4.43 \%)$ and mixed dentitions $(3.79 \%)$. Posterior teeth were the most related to odontogenic infections $(82 \%)$ compared with anterior teeth $(18 \%)$.

The involved teeth were recorded in all cases and the left mandibular third molar was most often related to the odontogenic infection (13.63\%), followed by the left mandibular first molar (11.3\%), 48 (7.14\%) and left mandibular second molar $(6.49 \%)$.

Regarding the side of the facial involvement it was verified that the left side was most frequently affected $(56.75 \%)$, followed by the right side $(32.97 \%)$ and both sides at the same time $(10.27 \%)$. Evaluating the facial spaces affected and the respective tooth, it was found that the submandibular space was the most affected due to the left mandibular third and first molar (Table 1). The canine space (10 cases) and the oral vestibulum space ( 8 cases) were the most affected when the infections were from the anterior teeth (Table 2). The oral space (5 cases) was most commonly involved when the infection originated from the premolars, followed by the canine and submandibular spaces with 3 cases each (Table 3). Usually the deciduous teeth were related to oral vestibulum region infections (Table 4).

About half of the evaluated patients presented very bad oral hygiene during the initial clinical examination $(50.63 \%)$, $18.98 \%$ of the patients presented regular oral hygiene and only $22.78 \%$ presented good oral hygiene. Dental decay was the main etiologic factor for the odontogenic infections $(90.90 \%)$, followed by unsatisfactory root canal treatments 
Table 1. Facial spaces affected distribution and respective posterior teeth that were the cause of the infection.

\begin{tabular}{|c|c|c|c|c|c|c|c|c|c|c|c|c|c|}
\hline \multirow[t]{2}{*}{ FACIAL SPACES } & \multicolumn{9}{|c|}{ TEETH * } & \multirow[b]{2}{*}{46} & \multirow[b]{2}{*}{47} & \multirow[b]{2}{*}{48} & \multirow[t]{2}{*}{ TOTAL } \\
\hline & 16 & 17 & 18 & 26 & 27 & 28 & 36 & 37 & 38 & & & & \\
\hline Submandibular & 0 & 0 & 0 & 0 & 0 & 0 & 8 & 3 & 9 & 4 & 6 & 5 & 35 \\
\hline Buccal & 2 & 0 & 0 & 3 & 1 & 2 & 2 & 3 & 0 & 3 & 3 & 1 & 20 \\
\hline Sublingual & 0 & 0 & 0 & 0 & 0 & 0 & 1 & 1 & 0 & 0 & 0 & 0 & 2 \\
\hline Sublingual + Submandibular & 0 & 0 & 0 & 0 & 0 & 0 & 1 & 1 & 1 & 0 & 0 & 1 & 4 \\
\hline Parapharyngeal + Submandibular & 0 & 0 & 0 & 0 & 0 & 0 & 1 & 0 & 1 & 0 & 0 & 1 & 3 \\
\hline Submandibular+Buccal+Submental+Sublingual & 0 & 0 & 0 & 0 & 0 & 0 & 1 & 0 & 0 & 0 & 0 & 0 & 1 \\
\hline Paranasal sinuses+Orbital & 0 & 0 & 0 & 1 & 1 & 0 & 0 & 0 & 0 & 0 & 0 & 0 & 2 \\
\hline Submandibular+Submental+Sublingual & 0 & 0 & 0 & 0 & 0 & 0 & 0 & 0 & 1 & 0 & 0 & 0 & 1 \\
\hline Retropharyngeal & 0 & 0 & 0 & 0 & 0 & 0 & 0 & 0 & 1 & 0 & 0 & 0 & 1 \\
\hline Subperiosteal & 0 & 0 & 0 & 0 & 0 & 0 & 0 & 0 & 1 & 0 & 0 & 0 & 1 \\
\hline Retropharyngeal + Submandibular & 0 & 0 & 0 & 0 & 0 & 0 & 0 & 0 & 1 & 0 & 0 & 0 & 1 \\
\hline Parapharyngeal + Submandibular+ Buccal & 0 & 0 & 0 & 0 & 0 & 0 & 0 & 1 & 0 & 0 & 0 & 1 & 2 \\
\hline Oral vestibule & 1 & 0 & 0 & 0 & 0 & 0 & 1 & 0 & 1 & 0 & 0 & 0 & 3 \\
\hline TOTAL & 3 & 0 & 0 & 4 & 2 & 2 & 15 & 9 & 16 & 7 & 9 & 9 & 76 \\
\hline
\end{tabular}

* - 16 - Right maxillary first molar; 17 - Right maxillary second molar; 18 - Right maxillary third molar; 26 - Left maxillary first molar; 27 - left Maxillary second molar 28 - left maxillary third molar; 36 - left mandibular first molar; 37 - left mandibular second molar; 38 - left mandibular third molar; 46 - right mandibular first molar; 47 right mandibular second molar; 48 - right mandibular third molar.

Table 2. Facial spaces affected distribution and the respective anterior teeth that was the cause of the infection.

\begin{tabular}{|c|c|c|c|c|c|c|c|c|c|c|c|c|c|}
\hline \multirow[t]{2}{*}{ FACIAL SPACES } & \multicolumn{12}{|c|}{ TEETH * } & \multirow[t]{2}{*}{ TOTAL } \\
\hline & 11 & 12 & 13 & 21 & 22 & 23 & 31 & 32 & 33 & 41 & 42 & 43 & \\
\hline Canine & 0 & 1 & 5 & 0 & 0 & 4 & 0 & 0 & 0 & 0 & 0 & 0 & 10 \\
\hline Submandibular & 0 & 0 & 0 & 0 & 0 & 0 & 0 & 0 & 0 & 0 & 0 & 1 & 1 \\
\hline Sublingual & 0 & 0 & 0 & 0 & 0 & 0 & 0 & 2 & 0 & 0 & 0 & 0 & 2 \\
\hline Submandibular+Submental+Sublingual & 0 & 0 & 0 & 0 & 0 & 0 & 0 & 0 & 1 & 0 & 0 & 1 & 2 \\
\hline Canine + Buccal & 1 & 0 & 1 & 0 & 0 & 0 & 0 & 0 & 0 & 0 & 0 & 0 & 2 \\
\hline Parapharyngeal + Submandibular+ Buccal & 0 & 0 & 0 & 0 & 0 & 0 & 0 & 0 & 0 & 1 & 0 & 0 & 1 \\
\hline Oral vestibule & 1 & 1 & 0 & 2 & 1 & 1 & 0 & 0 & 1 & 0 & 0 & 1 & 8 \\
\hline TOTAL & 2 & 2 & 6 & 2 & 1 & 5 & 0 & 2 & 2 & 1 & 0 & 3 & 26 \\
\hline
\end{tabular}

* - 11 - Right maxillary central incisor. 12 - right maxillary lateral incisor; 13 - right maxillary canine; 21 - left maxillary central incisor; 22 - left maxillary lateral incisor; 23 - left maxillary canine; 31 - left mandibular central incisor; 32 - left mandibular lateral incisor; 33 - left mandibular canine; 41 - right mandibular central incisor; 42 right mandibular lateral incisor; 43 - left maxillary canine.

Table 3. Facial spaces affected distribution and the respective premolars that was the cause of the infection.

\begin{tabular}{lllllllll|c}
\hline FACIAL SPACES & & & & & TEETH * & & TOTAL \\
Submental & 14 & 15 & 24 & 25 & 34 & 35 & 44 & 45 & \\
Canine & 0 & 0 & 0 & 0 & 1 & 0 & 0 & 0 & 1 \\
Submandibular & 0 & 0 & 3 & 0 & 0 & 0 & 0 & 0 & 3 \\
Buccal & 0 & 0 & 0 & 0 & 1 & 0 & 1 & 1 & 3 \\
Sublingual & 0 & 1 & 0 & 2 & 0 & 2 & 0 & 0 & 5 \\
Canine + Buccal & 1 & 0 & 0 & 0 & 1 & 0 & 1 & 0 & 2 \\
Oral vestibule & 1 & 0 & 0 & 0 & 0 & 0 & 0 & 0 & 1 \\
\hline TOTAL & 2 & 1 & 3 & 2 & 3 & 3 & 2 & 1 & 17 \\
\hline
\end{tabular}

* - 14 - Right maxillary first premolar; 15 - right maxillary second premolar; 24 - left maxillary first premolar; 25 - left maxillary second premolar; 34 - left mandibular first premolar; 35 - left mandibular second premolar; 44 - right mandibular first premolar; 45 - right mandibular second premolar.

$(4.54 \%)$, pericoronitis $(3.24 \%)$ and post-extraction complications (1.29\%) (Figure 1).

Surgical treatment was used in $76 \%$ of the cases and nonsurgical in $24 \%$. From all the surgically treated patients, $63 \%$ had the procedures done under local anesthesia and $37 \%$ required general anesthesia. The patients managed with general anesthesia were hospitalized for 3.52 days in average, with 2.90 days from the surgery to the hospital dismissal The follow-up of these patients was in average 30 days.

Relative to the drain, penrose drain number 1 was used in $38 \%$ of the cases. In $33 \%$ of the surgically managed patients no drains were used, and the association of penrose 
Table 4. Facial space distribution and the respective deciduous teeth that was the cause of the infection.

\begin{tabular}{llllll|c}
\hline FACIAL SPACES & $\mathbf{5}$ & $\mathbf{8 4}$ & $\mathbf{5 1}$ & $\mathbf{6 4}$ & $\mathbf{7 4}$ & TOTAL \\
& $\mathbf{5 4}$ & 1 & 0 & 0 & 0 & 2 \\
Buccal & 1 & 0 & 1 & 3 & 1 & 5 \\
\hline Oral vestibule & 0 & 1 & 1 & 3 & 1 & 7 \\
\hline Total & 1 & 1 &
\end{tabular}

* - 54 - Right maxillary first decidous molar; 84 - right mandibular first deciduous molar; 51 right maxillary deciduous central incisor; 64 - left maxillary first deciduous molar; 74 - left mandibular deciduous first molar.

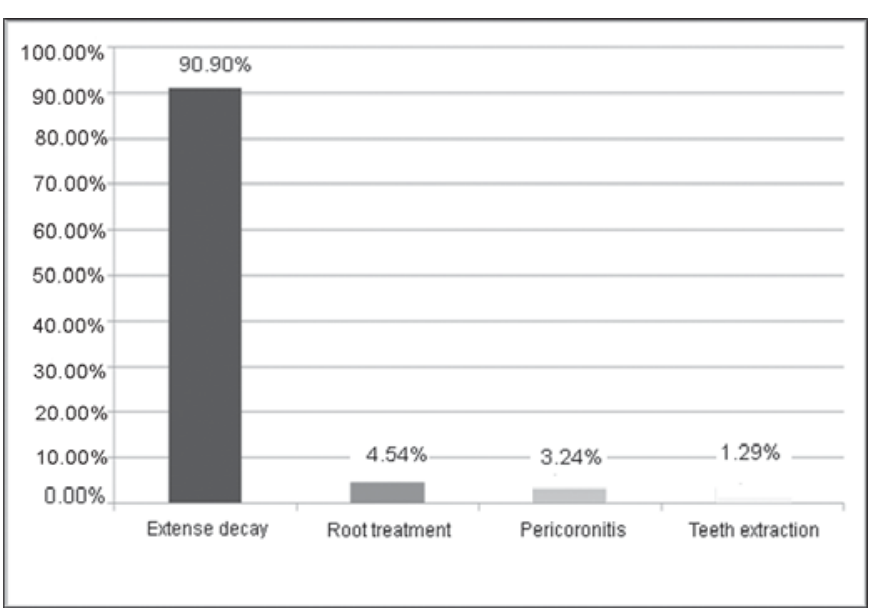

Fig. 1 - Study etiology distribution.

drain and irrigation tubes (number 12) was used in $29 \%$ of the cases. That association was used in the most severe cases where saline irrigation through the drains was required and it was removed as soon as the secretion diminished.

Some patients reported paresthesia, and out of them $60 \%$ complained about the mentual nerve area and $40 \%$ about the infraorbital area; none of the patients complained of long lasting paresthesia.

The association of amoxicillin with clavulanate was the more often applied antibiotic drug therapy (29.74\%), followed by the association of cephalexin and metronidazole (25.94\%) and amoxicillin and metronidazole (15.82\%); other associations or isolated antibiotics were applied in some cases $(15.82 \%)$.

In this sample there was only one case in which reintervention was necessary for a new surgical incision and drainage of the facial abscess. Complications like dehiscence, need for drug change and hypertrophic scar or even keloid at the incision site, were not observed in this study.

\section{Discussion}

In this study, men were more affected (53.33\%), which is in agreement with the findings in the scientific literature. Although the reason is unclear, Dodson et al. (1989) ${ }^{10}$ reported that the male gender has greater tendency to suffer facial and neck traumas, which could lead to an exacerbation of chronic processes. They usually have a worse hygiene condition than women, beyond the fact that men neglect more often light infections in the mouth and face.

Indresano et al. (1992) $)^{11}$ has estimated that $80 \%$ of the population have at least one third molar and they are commonly associated to complications such as pain, swelling and decay, being one of the probable causes of deep facial space infections. In this study it was verified that the posterior teeth were the main responsible for odontogenic infections $(82 \%)$, especially the posterior mandibular teeth, among which the left mandibular third molar was the most affected $(13.6 \%)$. This is probably due to the higher difficulty in brushing and cleaning the posterior teeth. Decay was the cause for $90.9 \%$ of the odontogenic infections, as previously described by Flynn et al. (2006) ${ }^{12-13}$ who reported it as the main etiologic factor $(65 \%)$.

Another reason for the number of decayed teeth could be a social problem found in this country. Amaral et al. $(2014)^{14}$ reported presence of $58.6 \%$ decayed teeth in a study that evaluated 303 children in a school of a São Paulo state city; Xavier et al. (2012) ${ }^{15}$ also state the need of planning educational activities and adoption of preventive policy measures to change this reality.

Rega et al. (2006) ${ }^{16}$ reported that the submandibular space was the most often affected in cases of facial infection. In the present study this same facial space was the most affected (30.3\%). According to Dodson et al. (1991) $)^{5}$ and Schuknecht et al. $(2008)^{17}$ the origin and localization of the infection are strongly related because the abscess origin defines its localization as well as the localization defines its origin.

Thirty seven percent of the patients were submitted to general anesthesia for the surgical treatment in this sample. The authors agree with the hospitalization criteria for patients who present facial infections described by Flynn et al. $(2006)^{12-13}$. According to this criterion the patients selected for hospital admission are those who presented big swelling in one or more deep facial or neck spaces that could be lifethreatening due to the proximity of vital structures and/or airway obstruction; body temperature over $38.5{ }^{\circ} \mathrm{C}$; need of previous concomitant systemic disease control and need of surgical treatment under general anesthesia.

The hospitalization period was in average of 3.52 days (time between the admission and the discharge), a period quite similar to the one found by Krishnan et al. (1993) ${ }^{1}$ which was 4 days; and not similar to the results described by Indresano et al. (1992) ${ }^{11}$ who presented an average of 6.2

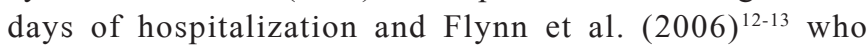
reported an average of 5.1 days with a standard deviation of \pm 3. Indresano et al. (1992) $)^{11}$ and Wang et al. (2005) $)^{2}$ state that long-term hospitalization could become a negative factor for the patient and the society. 
According to Peterson et al. $(2002)^{3}$ and Miloro et al. $(2011)^{18}$ the main objectives of the facial infection treatment are: pain relief, function recovery, vital structure preservation, to prevent flare or relapses and to limit the incapacity period. In the present study the surgical treatment was performed in $76 \%$ of the cases and was made in order to extinguish the etiologic agent, puncture and aspiration of the affected area (for secretion examination by culture and antibiogram), cellulitis and facial abscess incision and drainage, necrotic tissues removal, abundant saline irrigation, drains and irrigation tubes insertion and daily dressing. Nonsurgical treatment was adopted in $24 \%$ of the total cases, usually where the infection was chronic and limited.

All patients included in this study had complete resolution of the infectious disease and perfect reestablishment of the affected tissues. This agrees with Wang et al. (2005) ${ }^{2}$ who stated that microbiologic culture and antibiogram do not seem to be clinically useful; probably because the surgical treatment performed adequately and the previous knowledge of the microbiology involved in facial and oral infection sites lead to an empiric choice of antibiotic medication that could be employed with a considerable confidence level ${ }^{18}$.

According to Peterson (2002) ${ }^{3}$ and Miloro et al. (2011) ${ }^{18}$ the antibiotic medication choice must follow four criteria. First, the antibiotic must be effective against all the microorganisms that are usually responsible for facial infections. Second, the medication should have a limited spectrum, when possible, in order not to interfere on the normal microbiota of the patient. Third, the antibiotic must be as less toxic as possible and fourth, the drug must be bactericidal, because the patient affected by facial infection could be defenseless and the bacteriostatic antibiotics could lead to a slow recovery. Rega et al. $(2006)^{16}$ state that the penicillins still are the empiric first choice antibiotic for odontogenic infections, due to their effectiveness, minimal side effects, low cost, good patient tolerance and easy accessibility.

Other authors state that basic beta lactum antibiotics are key antibiotics to start treating odontogenic infections, as these infections are predominantly of gram-positive aerobes $^{19-20}$. On the other hand, some authors ${ }^{21}$ described the organisms causing infection as aerobes $(68.2 \%)$, mixed $(13.6 \%)$ and anaerobes $(9.1 \%)$, and they state that according to their results the microflora-causing maxillofacial infection did not change, and penicillin remains the drug of choice in treating these infections ${ }^{21}$.

Only in one case the reintervention was needed in this sample, and it was the single complication found by the authors in this study. It was the case of a woman (53 years old) who used chronic steroids, and probably had the organic defense capacity decreased due to the immunosuppressive properties of the drug. Additionally, for infections with special difficulty to treat, fungal contamination should be investigated ${ }^{22}$.

Based on the results, the authors concluded that the facial infections should be promptly treated by proper antibiotic therapy and surgical management; and even without the results for culture and antibiogram the authors achieved success in all facial infections treated.

\section{Acknowledgements}

FAPESP for the financial support to this study $\left(\mathrm{N}^{\circ} 2010 /\right.$ 09430-0).

\section{References}

1. Krishnan V, Johnson JV, Helfrick JF. Management of maxillofacial infections: a review of 50 cases. J Oral Maxillofac Surg. 1993; 51: 868-73.

2. Wang J, AhaniA, Pogrel MA. A five-year retrospective study of odontogenic maxillofacial infections in a large urban public hospital. Int J Oral Maxillofac Surg. 2005; 34: 646-9.

3. Peterson LJ, Ellis III E, Hupp JR, Tucker MR. Contemporary oral and maxillofacial surgery. Saint Louis: Mosby; 2002. p.343-79.

4. Maestre VJR. Treatment options in odontogenic infection. Med Oral Patol Oral Cir Bucal. 2004; 9(Suppl): S19-31.

5. Dodson TB, Barton JA, Kaban LB. Predictors of outcome in children hospitalized with maxillofacial infections: a linear logistic model. J Oral Maxillofac Surg. 1991; 49: 838-42.

6. Sánchez R, Mirada E, Arias J, Paño JR, Burqueño M. Severe odontogenic infections: epidemiological, microbiological and therapeutic factors. Med Oral Patol Oral Cir Bucal. 2011; 16: e670-6.

7. McKellop JA, Bou-Assaly W, Mukherji SK. Emergency head \& neck imaging: infections and inflammatory processes. Neuroimag Clin N Am. 2010; 20: 651-61.

8. Mathew GC, Ranganathan LK, Gandhi S, Jacob ME, Singh I, Solanki M, et al. Odontogenic maxillofacial space infections at a tertiary care center in North India: a five-year retrospective study. Int J Infect Dis. 2012; 16: e296-302.

9. Sato FRL, Hajala FAC, Freire Filho FWV, Moreira RWF, Moraes M. Eightyear retrospective study of odontogenic origin infections in a postgraduation program in oral and maxillofacial surgery. J Oral Maxillofac Surg. 2009; 67 : 1092-7.

10. Dodson TB, Perrott DH, Kaban LB. Pediatric maxillofacial infections: a retrospective study of 113 patients. J Oral Maxillofac Surg. 1989; 47: 327-30.

11. Indresano AT, Haug RH, Hoffman MJ. The third molar as a cause of deep space infections. J Oral Maxillofac Surg. 1992; 50: 33-5.

12. Flynn TR, Shanti RM, Levi MH, Adamo AK, Kraut RA, Trieger N. Severe odontogenic infections, Part 1: Prospective report. J Oral Maxillofac Surg. 2006; 64: 1093-103.

13. Flynn TR, Shanti RM, Hayes C. Severe odontogenic infections, Part 2 : prospective outcomes study. J Oral Maxillofac Surg. 2006; 64: 1104-13.

14. Amaral RC, Batista MJ, Meirelles MPMR, Cypriano S, Sousa MLR Dental caries trends among preschool children in Indaiatuba, SP. Brazil. Braz J Oral Sci. 2014; 13: 1-5.

15. Xavier A, Carvalho FS, Bastos RS, Caldana ML, Bastos JRM. Dental caries-related quality of life and socioeconomic status of preschool children, Bauru, SP. Braz J Oral Sci. 2012; 11: 463-8.

16. Rega AJ, Aziz SR, Ziccardi VB. Microbiology and antibiotic sensitivities of head and neck space infections of odontogenic origin. J Oral Maxillofac Surg. 2006; 64: 1377-80.

17. Schuknecht B, Stergiou G, Graetz K. Masticator space abscess derived from odontogenic infection: imaging manifestation and pathways of extension depicted by CT and MR in 30 patients. Eur Radiol. 2008;18: 1972-9.

18. Miloro M, Ghali GE, Larsen $P$, Waite P. Peterson's principles of oral and maxillofacial surgery. Shelton: PMPH-USA; 2011. p.841-61.

19. Kuriyama T, Nakagawa K, Karasawa T, Saiki Y, Yamamoto E, Nakamura S. Past administration of beta-lactam antibiotics and increase in the emergence of beta-lactamase-producing bacteria in patients with orofacial odontogenic infection. Oral Surg Oral Med Oral Pathol Oral Radiol Endod. 2000; 89: 186-92. 
20. Walia IS, Borle RM, Mehendiratta D, Yadav AO. Microbiology and antibiotic sensitivity of head and neck space infections of odontogenic origin. J Maxillofac Oral Surg. 2014; 13: 16-21.

21. Yuvaraj $\mathrm{V}$, Alexander M, Pasupathy S. Microflora in maxillofacial infection - A changing scenario? J Oral Maxillofac Surg. 2012; 70: 119-25.

22. Almeida OP, Scully C. Fungal infections of the mouth. Braz J Oral Sci. 2002; 1: 19-26. 